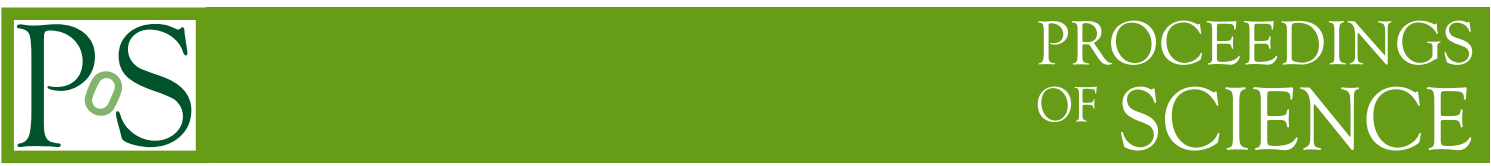

\title{
Very High Energy Emission from Gamma-Ray Bursts
}

\section{Soebur Razzaque*}

Dept. of Physics, University of Johannesburg, PO Box 524, Auckland Park 2006, South Africa

E-mail: srazzaque@uj.ac.za

\section{Reetanjali Moharana}

Dept. of Physics, University of Johannesburg, PO Box 524, Auckland Park 2006, South Africa E-mail: reetanjalim@uj.ac.za

Gamma-Ray Bursts (GRBs), the most powerful sources of gamma rays in the universe, have been detected at energies up to $95 \mathrm{GeV}$. This energy is at the verge of what is known as the Very High Energy (VHE, above $100 \mathrm{GeV}$ ) emission regime. VHE sources are targets for currently running and upcoming ground-based Cherenkov telescopes. It is therefore very important to understand the VHE emission mechanism(s) of GRBs. Synchrotron radiation by electrons accelerated in the external shocks of a GRB blast wave, widely accepted as the mechanism for X-ray to radio afterglow emission, has difficulty to explain $\gtrsim 10 \mathrm{GeV}$ emission hours after the GRB trigger. We model VHE emission from interactions of cosmic-rays which are shock-accelerated in the GRB blast wave. We also compute VHE emission from electron-positron pair cascade formed in the intergalactic space by absorption of $\gtrsim 1 \mathrm{PeV}$ gamma rays from the prompt phase of the GRB. VHE emissions from the GRBS modeled here can be tested by the ground-based air and water Cherenkov telescopes.

The 34th International Cosmic Ray Conference,

30 July- 6 August, 2015

The Hague, The Netherlands

\footnotetext{
* Speaker.
} 


\section{Introduction}

GRBs are the most powerful explosions in the universe with most of their visible energy emitted in $\sim$ 0.1-1 MeV gamma rays [1]. The Energetic Gamma Ray Experiment Telescope (EGRET) onboard the Compton Gamma Ray Observatory (CGRO) detected up to $18 \mathrm{GeV}$ gamma rays from a handful of GRBs [2], often with a significant time delay after the prompt emission. This timelag and a plausible hard component in the $\mathrm{GeV}$ range, inferred from EGRET data, are now confirmed by the Large Area Telescope (LAT) onboard the Fermi Gamma-Ray Space Telescope [3]. Fermi-LAT detects roughly $\sim 9 \%$ of the bursts triggered by the Gamma-ray Burst Monitor (GBM) onboard Fermi which are in the common field of view of the two instruments. This is likely due to high fluence of the LAT-detected GRBs, for both long- and short-duration bursts, which are also intrinsically most energetic [4]. The low rate of Fermi-LAT GRB indicates that the GeV emission from GRBs is a fairly rare event. It remains to be observed VHE emission from GRBs and probe the nature of those bursts.

Detection of GRB 130427A is a bright spot in the history of the GRB science as it is the most comprehensibly studied GRB from high-energy gamma-rays down to radio frequencies $[5,6,7]$. At a redshift $z=0.34$, it is one of the brightest and most energetic GRBs ever detected. GeV emission from this burst lasted for a record 20 hours with detection of a $95 \mathrm{GeV}$ gamma ray $244 \mathrm{~s}$ after the trigger [5]. This VHE photon at the source frame gives some clues to the plausible VHE emitting GRBs and gives hope of detection by ground-based detectors and collect more statistics. Indeed,highly sensitive Imaging Atmospheric Cherenkov Teleescopes (IACTs) such as MAGIC, VERITAS and HESS-II with a threshold energy as low as $30 \mathrm{GeV}$ are currently slewing to the GRB directions, although no confirmed detection yet $[8,9,10]$. High Altitude Water Cherenkov (HAWC) detector was running at only $\sim 10 \%$ of its full capacity during the GRB 130427A and published a flux upper limit [11].

Simultaneous observations of GRBs by the Fermi LAT and Cherenkov telescopes in the overlapping GeV-VHE energy range will be extremely helpful to understand the nature of high-energy radiation. Such observations can also pose strong constraints on both the leptonic and/or hadronic models. Indeed detection of several $\gtrsim 10 \mathrm{GeV}$ photons very late in the afterglow of GRB 130427A challenges the electron-sychrotron model for their production [5]. Synchrotron-Self-Compton (SSC) emission $[12,13]$ is a favored mechanism for production of late high-energy photons, although no convincing evidence exists in GRB data yet. Hadronic emission mechanisms involve proton-synchrotron radiation $[14,15]$ and photohadronic interactions resulting in cascade gammaray production [16]. Cascade gamma-ray production in the Extragalactic Background Light (EBL) and Cosmic Microwave Background (CMB) in cosmic voids with very low $\left(\lesssim 10^{-18} \mathrm{G}\right)$ magnetic field can also lead to long-lasting VHE emission [17].

Apart from probing emission mechanism(s), detection of VHE from GRBs can be modeled to estimate the total explosion energy and test a radiative or adiabatic fireball model. This information has also implications for Gravitational Wave detection by Advanced LIGO/VIRGO. Detection of VHE emission from GRBs can be used to probe the EBL models and impose stringent constraints on quantum gravity models that lead to energy-dependent speed of light.

We present results from our ongoing work on modeling VHE emission from GRBs. In particular we discuss photohadronic interactions in the GRB blastwave and resulting cascade gamma-ray 
emission, as well as VHE emission from electron-positron pairs that are created from absorption of $\gtrsim 1 \mathrm{PeV}$ photons in the EBL and which inverse Compton scatter CMB photons. We take GRB 130427A as a particular example for calculations.

\section{VHE gamma rays from GRB afterglow}

VHE photons can be produced by decay of neutral pions from interactions of ultrahigh-energy protons in the GRB blast wave. Fermi accelerated high energy protons can interact with low energy photons to produce neutral pions and subsequently to high energy photons. We have shown the photon flux from pion decay in case of Adibatic wind environment, where the blast wave radius and bulk Lorentz factor evolve as

$$
\begin{aligned}
& R=1.4 \times 10^{17}(1+z)^{-1 / 2} A_{*}^{-1 / 2} E_{55}^{1 / 2} t_{2}^{1 / 2} \mathrm{~cm}, \\
& \Gamma=78(1+z)^{1 / 4} A_{*}^{-1 / 4} E_{55}^{1 / 4} t_{2}^{-1 / 4} .
\end{aligned}
$$

The typical spectral break energies for synchrotron radiation from electrons in the GRB blast wave evolution in wind can be written as [18]

$$
\begin{array}{r}
\varepsilon_{c}=h v_{c}=0.3 \times 10^{-9}(1+z)^{-3 / 2} \varepsilon_{B,-1}^{-3 / 2} A_{*}^{-2} E_{55}^{1 / 2} t_{2}^{1 / 2} \mathrm{GeV} \\
\varepsilon_{m}=h v_{m}=10 . \times 10^{-6}(1+z)^{1 / 2} \varepsilon_{B,-1}^{1 / 2} \varepsilon_{e, 0.1}^{2} E_{55}^{1 / 2} t_{2}^{-3 / 2} \mathrm{GeV} \\
\varepsilon_{s}=h v_{s}=1.8(1+z)^{-3 / 4} \phi_{1}^{-1} A_{*}^{-1 / 4} E_{55}^{1 / 4} t_{2}^{-1 / 4} \mathrm{GeV}
\end{array}
$$

where the maximum flux is

$$
F_{V, \max }=10.4(1+z)^{-1 / 2} \varepsilon_{b, 0.1}^{1 / 2} A_{*} E_{55}^{1 / 2} t_{2}^{-1 / 2} d_{28}^{-2} \mathrm{Jy} .
$$

We have done the VHE emission calculation for GRB 130427A with the following parameters, fractional magnetic energy density $\varepsilon_{b, 0.1}=0.025$, blast wave kinetic energy in $10^{55}$ erg unit $E_{55}=$ 0.5 , fractional electron energy density $\varepsilon_{e, 0.1}=0.095$, the wind parameter $A_{*}=0.1$, acceleration efficiency parameter $\phi_{1}=1$, redshift $z=0.34$ and distance $d=1331.6 \mathrm{Mpc}$. For the first $100 \mathrm{sec}$ the synchrotron spectrum is in the fast cooling regime and the spectrum is given by

$$
F_{v}=F_{v, \max }\left\{\begin{array}{lc}
\left(\frac{v_{c}^{\prime}}{v_{m}^{\prime}}\right)^{-3 / 2}\left(\frac{v^{\prime}}{v_{c}^{\prime}}\right)^{-2 / 3}\left(\frac{v^{\prime}}{v_{c}^{\prime}}\right) ; \quad v_{a}^{\prime}<v^{\prime}<v_{c}^{\prime} \\
\left(\frac{v_{m}^{\prime}}{v_{m}^{\prime}}\right)^{-1 / 2} ; & v_{c}^{\prime} \leq v^{\prime} \leq v_{m}^{\prime} \\
\left(\frac{v^{\prime}}{v_{m}^{\prime}}\right)^{-\frac{k}{2}} ; & v_{m}^{\prime}<v^{\prime}<v_{s}^{\prime}
\end{array}\right.
$$

At later time the synchrotron spectrum is in the slow cooling regime, and the flux is given by,

$$
F_{v}=F_{v, \max }\left\{\begin{array}{l}
\left(\frac{v}{v_{m}}\right)^{\frac{1}{3}} ; \quad v_{a}<v<v_{m}, \\
\left(\frac{v}{v_{m}}\right)^{\frac{-(k-1)}{2}} ; \quad v_{m}<v<v_{c} \\
\left(\frac{v_{c}}{v_{m}}\right)^{\frac{-(k-1)}{2}}\left(\frac{v}{v_{c}}\right)^{\frac{-k}{2}} ; \quad v<v_{s}
\end{array}\right.
$$



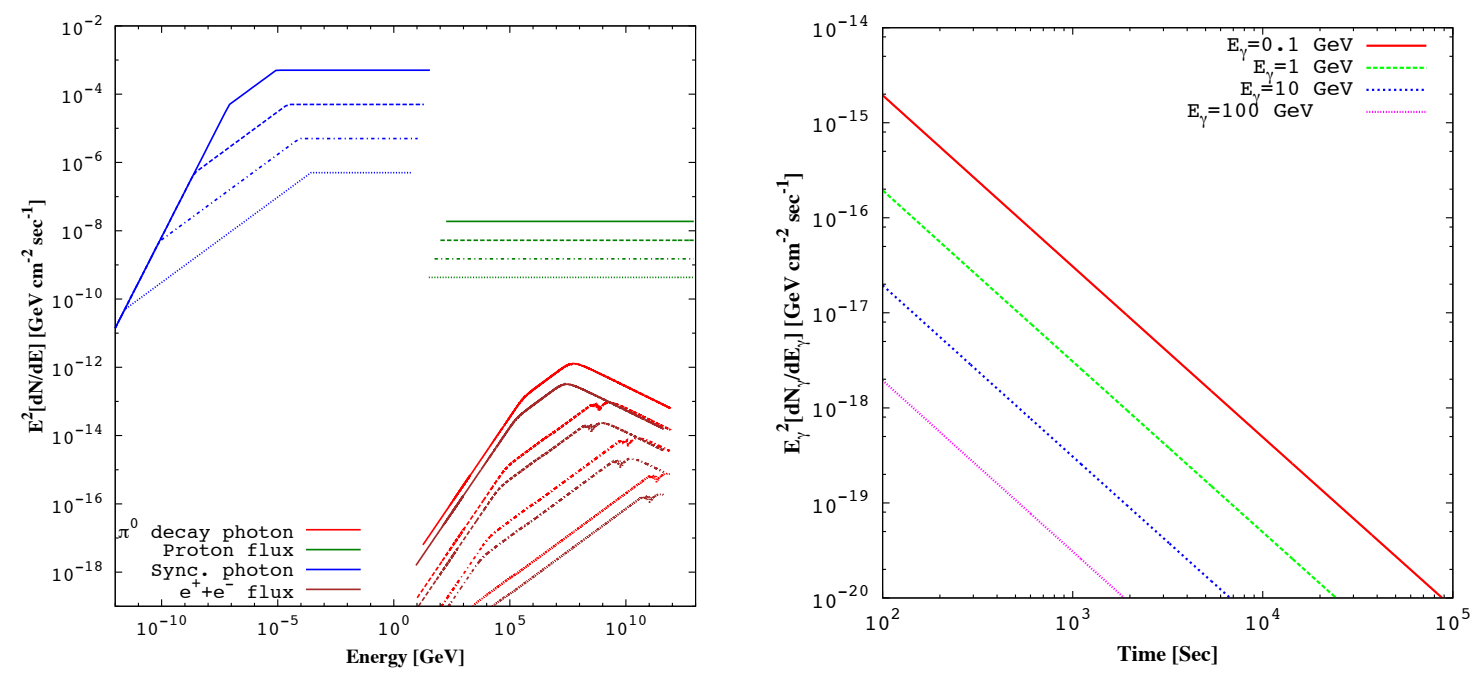

Figure 1: left panel: Flux of electron synchrotron photon (blue), cosmic proton flux (green), $\pi^{0}$ decay photon (red) flux and $\pi^{ \pm}$decay electron-positron flux (brown) from GRB 130427A blast wave is shown for time $10^{2}, 10^{3}, 10^{4}, 10^{5} \mathrm{sec}$ with solid, dashed,dot-dashed and dotted lines respectively. Right panel: Light curves for GRB 130427A at different energies.

Here we have taken $k=1.95$.

The cosmic ray proton flux is taken from ref. [18] and is given by

$$
E_{p, s}=6 \times 10^{9}(1+z)^{-5 / 4} \phi_{1}^{-1} A_{*}^{-1 / 4} \varepsilon_{b, 0.1}^{1 / 2} t_{2}^{1 / 4} E_{55}^{3 / 4} \mathrm{GeV}
$$

Using the opacity for $p \gamma$ interactions given in [18] we calculate the neutral pion flux as

$$
J_{\pi^{0}}\left(E_{\pi^{0}}\right) \sim \frac{1}{\langle x\rangle} J_{p}\left(\frac{E_{\pi}}{\langle x\rangle}\right) \tau_{P \gamma}\left(\frac{E_{\pi}(1+z)}{\Gamma\langle x\rangle}\right) .
$$

We have calculated the high energy photons produced from $\pi^{0} \rightarrow 2 \gamma$, and flux is given in Fig. 1, left panel. The energy spectrum of gamma-rays from pion decay is calculated by assuming each photon from $\pi^{0}$ decay caries half of its energy. Similarly $\pi^{ \pm}$decay $e^{ \pm}$fluxes have also been calculated in Fig. 1 assuming each electron or positron carries one-fourth the parent pion energy.

\section{Emission from cascades in intergalactic space by prompt photons}

Photons with energy up to the PeV range, escaping from the GRB fireball during the prompt phase can initiate an electron-positron pair cascade in the intergalactic space, which would produce secondary delayed emission [17]. Figure 2 shows simple geometry of such delayed emission. The delay arises because of deflection of electrons-positrons in the Inter-Galactic Magnetic Field (IGMF) and is given by

$$
\Delta t=\frac{1}{2}\left(t_{\mathrm{IC}}+\frac{1}{c} \lambda_{\gamma \gamma}\right) \theta_{L}^{2}
$$

Here the inverse-Compton cooling time for one interaction is $t_{\mathrm{IC}}=\lambda_{\mathrm{IC}} / c$ and the IC mean-free-path in CMB can be approximated as

$$
\lambda_{\mathrm{IC}}=\frac{0.71}{(1+z)^{4}}\left(\frac{\gamma_{e}}{10^{6}}\right)^{-1} \mathrm{Mpc} .
$$



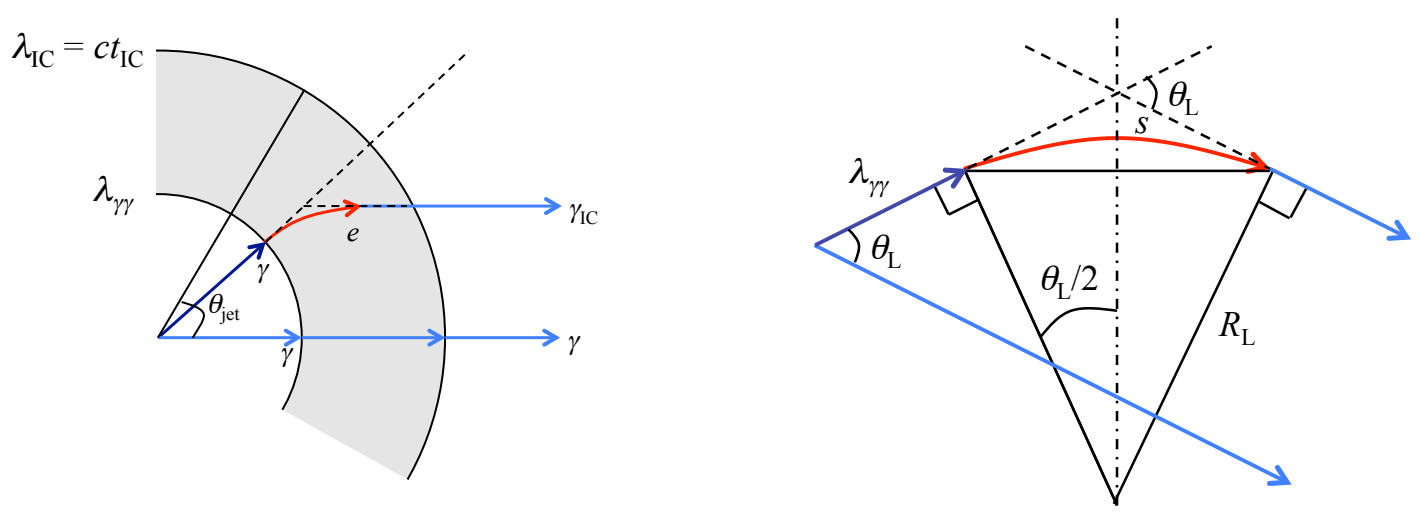

Figure 2: Simple geometry of the electron-positron cascade emission in the intergalactic space.

The deflection angle, $\theta_{L}=s / R_{L}$ for electrons-positrons in the IGMF is can be approximated as

$$
\theta_{L}=\frac{0.0013}{(1+z)^{4}}\left(\frac{B_{\mathrm{IGMF}}}{10^{-17} \mathrm{G}}\right)\left(\frac{\gamma_{e}}{10^{6}}\right)^{-2}
$$

with Larmor radius in the IGMF given by

$$
R_{L}=55.2\left(\frac{B_{\mathrm{IGMF}}}{10^{-17} \mathrm{G}}\right)^{-1}\left(\frac{\gamma_{e}}{10^{6}}\right) \mathrm{Mpc} .
$$

To calculate the delayed emission we assume the following simplifications [17]:

- Gamma rays are converted to electron-positron pairs by interacting with EBL photons close to the vicinity of the source. This is a good approximation for $E_{\gamma}(1+z) \gtrsim 20 \mathrm{GeV}$. As a result, no evolution of EBL is necessary.

- Explosive injection of electron-positron pairs in the intergalactic space. Since the observation time is much larger than the prompt GRB phase $(\sim 10 \mathrm{~s})$ it is a reasonable approximation.

- The inverse-Compton cooling length scale, $\lambda_{\mathrm{IC}}=c t_{\mathrm{IC}}$ for electron-positron pair in CMB is very short. As a result, no evolution of CMB is necessary.

- The inverse-Compton emission from electron-positron pairs is continuous and extended in time, over a duration $\Delta t_{\mathrm{obs}}$ due to their deflection in the IGMF.

For the primary prompt flux we take the hard component (with a photon index -1.7 ) in GRB 130427A between 11.5-33.0 s [5] and extending it up to $1 \mathrm{PeV}$. We calculate the delayed emission from the electron-positron pairs as [17]

$$
\frac{d N_{\text {delayed }}}{d E_{\mathrm{obs}, \mathrm{IC}} d t_{\mathrm{obs}} d A}=\int d \gamma_{e} \int d t_{s}\left[f\left(t_{s}\right) \frac{d N_{e}}{d \gamma_{e} d t_{s} d A}\right]\left[\frac{(1+z) t_{\mathrm{IC}}\left(\gamma_{e}\right)}{\Delta t_{\mathrm{obs}}\left(\gamma_{e}\right)}\right]\left[\frac{d N_{\mathrm{IC}}}{d E_{\mathrm{obs}, \mathrm{IC}} d t_{\mathrm{IC}}}\left(\gamma_{e}, \varepsilon_{\mathrm{cmb}}\right)\right],
$$

where $f\left(t_{s}\right) \approx T_{\mathrm{grb}} \delta\left(t_{s}\right)$ is the injection time for primary photons from the GRB prompt emission. The first term in brackets in equation (3.5) is the source term for electron-positron pairs given by,

$$
\frac{d N_{e}}{d \gamma_{e} d t_{s} d A}=\frac{1+z}{\gamma_{e}} F_{E}^{\mathrm{grb}}\left(1-\exp \left[\tau_{\gamma \gamma}(E, z)\right]\right)
$$



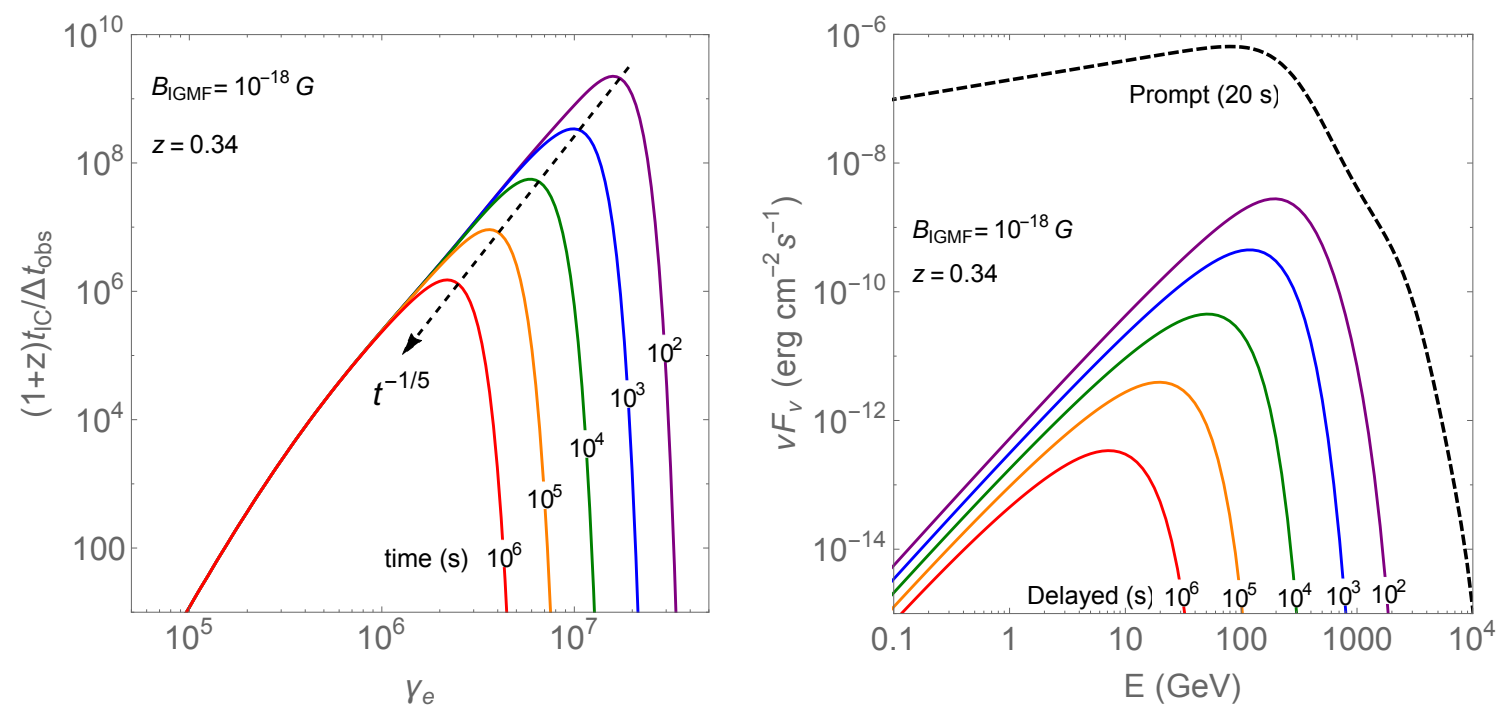

Figure 3: Left panel: Ratio of IC cooling time in CMB to the observed delayed time due to deflection in magnetic field for electrons of different Lorentz factor $\gamma_{e}$. Note that the peak of the $\gamma_{e}$ distribution scales as $t^{-1 / 5}$. Right panel: Prompt and delayed Inverse Compton flux from pair cascade in the intergalactic space.

where $E=2 \gamma_{e} m_{e} c^{2} /(1+z)$. The second term in brackets in equation (3.5) is the delay term which is plotted in Fig. 3, left panel. The peak of this ratio scales as $t^{-1 / 5}$ as shown in the plot. The observation time can be approximated as

$$
\Delta t_{\mathrm{obs}} \approx\left[\frac{8.4 \times 10^{10}}{(1+z)^{3}} \frac{\lambda_{\gamma \gamma}\left(\gamma_{e}, z\right)}{10 \mathrm{Mpc}}+\frac{6.1 \times 10^{9}}{(1+z)^{11}}\right]\left(\frac{B_{\mathrm{IGMF}}}{10^{-17} \mathrm{G}}\right)^{2}\left(\frac{\gamma_{e}}{10^{6}}\right)^{-5} \mathrm{~s} .
$$

The third term in equation (3.5) is the inverse-Compton kernel which we simplfy in the Thomson regime as

$$
\frac{d N_{\mathrm{IC}}}{d E_{\mathrm{obs}, \mathrm{IC}} d t_{\mathrm{IC}}}\left(\gamma_{e}, \varepsilon_{\mathrm{cmb}}\right) \approx(1+z) \frac{3 c \sigma_{T}}{4 \gamma_{e}^{2}} \frac{d n_{\mathrm{cmb}, \mathrm{pk}}}{d \varepsilon_{\mathrm{cmb}, \mathrm{pk}}}\left(1-\frac{E_{\mathrm{obs}, \mathrm{IC}}(1+z)}{4 \gamma_{e}^{2} \varepsilon_{\mathrm{cmb}, \mathrm{pk}}}\right),
$$

where $\varepsilon_{\mathrm{cmb}, \mathrm{pk}}$ is the CMB photon energy at the peak.

Figure 3, right panel, shows the delayed VHE emission from electron-positron pairs in CMB that are created by interactions of prompt GRB 130427A photons with EBL photons. We use the EBL model in refs $[19,20]$.

\section{Summary and Outlook}

We have calculated VHE emission from $p \gamma$ interactions in the GRB blast wave taking GRB 130427A as an example. We have also calculated VHE emission from the electron-positron pair cascade in the the intergalactic space. Our preliminary results show that VHE emission at early time for GRB blast wave evolution can be significant. Pair cascade emission can be potentially detected by the ground-based Cherenkov detectors.

\section{References}

[1] Gehrels, N., \& Razzaque, S. 2013, Frontiers of Physics, 8, 661 
[2] Hurley, K., Dingus, B. L., Mukherjee, R., et al. 1994, Nature, 372, 652

[3] Ackermann, M., Ajello, M., Asano, K., et al. 2013, Astrophys. J. Supp., 209, 11

[4] Cenko, S. B., Frail, D. A., Harrison, F. A., et al. 2011, Astrophys. J., 732, 29

[5] Ackermann, M., Ajello, M., Asano, K., et al. 2014, Science, 343, 42

[6] Preece, R., Burgess, J. M., von Kienlin, A., et al. 2014, Science, 343, 51

[7] Perley, D. A., Cenko, S. B., Corsi, A., et al. 2014, Astrophys. J., 781, 37

[8] Aleksić, J., Ansoldi, S., Antonelli, L. A., et al. 2014, MNRAS, 437, 3103

[9] Acciari, V. A., Aliu, E., Arlen, T., et al. 2011, Astrophys. J., 743, 62

[10] H.E.S.S. Collaboration, Abramowski, A., Aharonian, F., et al. 2014, A\&A, 565, A16

[11] Abeysekara, A. U., Alfaro, R., Alvarez, C., et al. 2015, Astrophys. J., 800, 78

[12] Chiang, J., \& Dermer, C. D. 1999, Astrophys. J., 512, 699

[13] Zhang, B., \& Mészáros, P. 2001, Astrophys. J., 559, 110

[14] Razzaque, S., Dermer, C. D., \& Finke, J. D. 2010, The Open Astronomy Journal, 3, 150

[15] Razzaque, S. 2010, Astrophys. J. Lett., 724, L109

[16] Böttcher, M., \& Dermer, C. D. 1998, Astrophys. J. Lett., 499, L131

[17] Razzaque, S., Mészáros, P., \& Zhang, B. 2004, Astrophys. J., 613, 1072

[18] Razzaque, S. 2013, Phys. Rev. D, 88, 103003

[19] Razzaque, S., Dermer, C. D., \& Finke, J. D. 2009, Astrophys. J., 697, 483

[20] Finke, J. D., Razzaque, S., \& Dermer, C. D. 2010, Astrophys. J., 712, 238 